\title{
Relativistic outflows from compact galactic sources
}

\author{
Pol Bordas* \\ Departament de Física Quàntica i Astrofísica, Institut de Ciències del Cosmos, Universitat de \\ Barcelona, IEEC-UB, Martí i Franquès 1, 08028 Barcelona, Spain \\ E-mail: pbordas afqa.ub . edu
}

\begin{abstract}
Relativistic outflows in the form of winds and jet-like features are ubiquitous phenomena present in a variety of galactic sources. These outflows can carry a significant fraction of the system's energy reservoir up to distances of a few tens of parsecs. Particle acceleration along the jets or at the jet or wind interaction sites with the surrounding medium leads to the production of copious non-thermal emission, which is observed in a broad energy range, from radio to very-highenergy gamma-rays. While powerful galactic jets are typically associated to accretion processes in BH/NS X-ray binaries, jet-like features have recently been imaged also from isolated systems, most notably from young pulsars moving at high-speeds through the interstellar medium. In this contribution I will review recent findings related to some of the most extreme examples of outflows from compact galactic sources, discussing the implications that these findings have in our understanding of the outflow formation and propagation mechanisms.
\end{abstract}

High Energy Phenomena in Relativistic Outflows VII - HEPRO VII

9-12 July 2019

Facultat de Física, Universitat de Barcelona, Spain

\footnotetext{
${ }^{*}$ Speaker.

${ }^{\dagger}$ Pol Bordas acknowledges the financial support by the Beatriu de Pinós Research Program from the Agency for Management of University and Research Grants (AGAUR) of the Generalitat de Catalunya. Pol Bordas acknowledges the research facilities provided by the Insitut de Ciències del Cosmos (ICCUB) and the Department of Quantum Physics and Astrophysics of the Universitat de Barcelona.
} 


\section{Introduction}

Gamma-ray astronomy has been providing in the last years with a wealth of observational results which have made of it a new window for the study of the non-thermal Universe. Several catalogues at high- and very-high energy gamma-rays (HEs: $100 \mathrm{MeV}<E_{\gamma}<100 \mathrm{GeV}$; VHEs: $E_{\gamma}>100 \mathrm{GeV}$ ) have been recently released by the Fermi-LAT and the H.E.S.S. and HAWC collaborations, respectively, demonstrating the power of gamma-ray astronomy and the whole new perspectives that gamma-ray astrophysics can bring, in particular for high-energy sources in our Galaxy. Intriguingly, the nature of these gamma-ray emitters has been established for just a fraction of the catalogued sources, thanks to either robust identifications based on some observational properties (e.g. flux variability,) or its positional or morphological association with lower-energy catalogued sources. In the vast majority of identified sources, and most likely also for the unidentified ones, gamma-rays originate from the vicinity of a compact object (black holes, neutron stars or white dwarfs) displaying powerful outflows in the form of relativistic winds and jets. In these proceedings we will review some of the most extreme examples of gamma-ray emitters displaying relativistic outflows recently found in our Galaxy. Although we will mainly discuss gamma-ray emitting sources in this context, we will also make use of recent results obtained at lower energies (e.g. X-rays in the case of the runaway pulsar IGR J11014-6103 ). In the next Sections we will discuss some recent advances, both observational and theoretical, accomplished in the study of microquasars (Sect. 2), gamma-ray binaries (Sect. 3) and runaway pulsar wind nebulae (Sect. 4). For each case, we will focus correspondingly on the recent results obtained for the microquasar SS433 at HEs and VHEs (Sect. 2.1), the gamma-ray binary PSR B1259-63 (Sect. 3.1), and the runaway pulsar wind nebula IGR J11014-6103 (Sect. 4.1). A summary and concluding remarks are provided in (Sect. 5).

\section{Jets from microquasars}

Microquasars ( $\mu \mathrm{Qs}$ ) are X-ray binaries featuring relativistic jets, a nomenclature that follows their phenomenological analogy with Active Galactic Nuclei (AGN) (see Mirabel et al. 1992). Given that AGNs were known sources of $\gamma$-rays, $\mu \mathrm{Q}$ s have long predicted to be gamma-ray emitters as well. In addition, non-thermal synchrotron emission from $\mu \mathrm{Q}$ jets had been resolved at radio/IR energies in several systems, which requires the presence of highly energetic (accelerated) electrons in their jets. Otherwise, at least in two $\mu$ Qs (SS433, see Migliari et al. 2002, and 4U 1630 47, see Díaz Trigo et al. 2013; for this latter system, see however Díaz Trigo et al. (2014)), the presence of baryons has been reported following the detection of emission lines of highly ionised elements. Accounting for these relativistic particle populations together with the strong matter and radiation fields in which $\mu$ Qs are embedded, gamma-ray emission may be produced either through IC scattering off the radiation field from the companion star or through proton-proton interactions mechanisms when hadrons are present in their jets (see e.g. Romero et al. 2003; Dermer \& Böttcher 2006; Bosch-Ramon et al. 2006a). Large-scale $\gamma$-ray emission from $\mu \mathrm{Q}$ jets interacting with the surrounding medium has also been proposed (see e.g. Bosch-Ramon et al. 2005; Bordas et al. 2009). 
The detection of $\mu \mathrm{Qs}$ at gamma-ray energies had to wait nevertheless for the launch of the latest generation of gamma-ray satellites ${ }^{1}$. As of today, four $\mu \mathrm{Qs}$ have been found to be steady or transient HE gamma-ray emitters: Cyg X-3 (Tavani et al. 2009; Fermi LAT Collaboration et al. 2009), Cyg X-1(Sabatini et al. 2013; Malyshev et al. 2013; Zanin et al. 2016; Zdziarski et al. 2017), V404 Cyg (Loh et al. 2016) and SS433 (Bordas et al. 2015; Rasul et al. 2019; Xing et al. 2019). At VHEs, $\mu$ Qs have remained rather elusive. Apart from a tentative detection of Cyg X-1 reported by the MAGIC Collaboration (Albert et al. 2007), the search for VHE emission from other systems revealed unfruitful in the last years Saito et al. (2009); Aleksić et al. (2010); Archambault et al. (2013); H. E. S. S. Collaboration et al. (2018). Very recently, however, the HAWC Collaboration has reported on the detection of VHE emission from SS433 (Abeysekara et al. 2018; see below). Furthermore, It is worth noting that a $\mu \mathrm{Q}$ scenario has been proposed also for some gamma-ray binary systems, although the nature of the powering engine in these systems is yet unclear.

\subsection{Case study: SS433}

SS433 was the first binary system in which highly energetic jets were discovered (Abell \& Margon 1979), and was later classified as a Galactic $\mu \mathrm{Q}$. The system is composed of a 10-20 solar-mass black hole orbiting every $\sim 13$ days its A3-7 companion star, and is distinguished by a continuous supercritical accretion regime, with an accretion rate as high as $\sim 10^{-4}$ solar masses per year. The accretion disk feeds two mildly relativistic jets carrying a kinetic energy power at a level of $L_{\text {jet }} \gtrsim 10^{39} \mathrm{erg} \mathrm{s}^{-1}$ (Dubner et al. 1998), ejected at a velocity of $0.26 c$ and precessing every $\sim 162$ days. As stated above, SS433 represents also one of only two cases in which the presence of baryonic material in the jets has been established through the detection of lines of highly ionised heavy elements (Migliari et al. 2002). Most of SS433's jet kinetic power is delivered at the shock interface to the surrounding W50 nebula, a large supernova remnant, SNR G39.7-2.0. Radio to Xray emission originated in the jet/nebula interaction sites has been proposed (Safi-Harb \& Ögelman 1997), whereas high-energy gamma-ray radiation from these regions has also been predicted (Heinz \& Sunyaev 2002), peaking at energies of $\Gamma_{\text {jet }} m_{\mathrm{p}} c^{2}$, where $\Gamma_{\text {jet }}$ is the bulk motion jet Lorentz factor. Very-high-energy gamma-ray emission has also been proposed (Aharonian \& Atoyan 1998; BoschRamon et al. 2005; Bordas et al. 2009), under the assumption that particle acceleration at the jet termination shock extends to $\mathrm{TeV}$ energies.

Several groups have recently claimed the detection of SS433 in the HE and VHE gamma-ray domain. The first tentative association of a significant HE gamma-ray signal towards SS433 was announced in Bordas et al. (2015). The authors made use of $\sim 5$ years of archival Fermi-LAT observations on the source, finding a gamma-ray excess towards SS433/W50. The gamma-ray flux has no significant signatures of either orbital or precession variability in the analysed data-set. The spectral energy distribution (SED) displays a maximum at about $250 \mathrm{MeV}$, extending up to about $\sim 800 \mathrm{MeV}$. The authors considered the possibility that the observed emission is produced through proton-proton collisions at the SS433/W50 interaction regions (see e.g. Bordas 2009; Bordas et al. 2010). Other scenarios, although not favoured, could not be ruled out, including gamma-ray emission produced by relativistic electrons through IC or relativistic Bremsstrahlung processes. An

\footnotetext{
${ }^{1} \mu \mathrm{Q}$ s were claimed to be strong and variable VHE $\gamma$-ray sources in the 80 's, the most notable example being the case of Cyg X-3, although these detections resulted to be highly controversial, see e.g. Chardin \& Gerbier (1989).
} 
updated analysis by the same authors, but accounting for a somewhat larger data-set and making use of the improvements associated with the new "Pass 8" calibration of the LAT data archive (Bordas et al. 2017), resulted in similar conclusions in terms of the source SED, but with an improved positional accuracy of the gamma-ray excess with respect to the the location of SS433/W50. Recently, Xing et al. (2019) reported on the analysis of $\sim 10$ years of LAT observations on the source, showing that the excess emission falls towards the western jet lobes. Almost contemporaneously, Rasul et al. (2019) also reported the analysis of a $\sim 9$ years of LAT observations, yielding a detection of SS433 at a statistical significance of $13 \sigma$ in the 200 to $500 \mathrm{MeV}$ energy range. A temporal analysis of this data-set revealed in addition a marginal evidence $(2.9 \sigma)$ for modulation of SS433's gamma-ray flux with the precession period of the system. This prompted the authors to suggest that at least a fraction of the detected gamma-ray flux was originating close to the object rather than from the jet termination regions.

At VHEs, the MAGIC and H.E.S.S. Collaborations reported on the joint analysis of observations on SS433/W50 taken during periods in which the putative TeV signal may not be attenuated due to periodic eclipses or precession of the disk envelope surrounding the central parts of the binary system (see e.g. Reynoso et al. 2008). They also explored the east/west interaction regions with the W50 nebula. No evidence of VHE gamma-ray emission neither from the central binary nor from the interaction regions was found (MAGIC Collaboration et al. 2018). Very recently, however, the HAWC collaboration published the results of a long-term campaign on the source, which resulted in the detection of SS433/W50 at energies of at least $\sim 25 \mathrm{TeV}$ (Abeysekara et al. 2018). The emission is localised at the eastern/western interaction regions, without significant fluxes coming from the central system. These authors conclude that the broad-band emission, from radio to VHE gamma-rays, observed from these regions is consistent with a single population of very energetic electrons embedded in a nebular magnetic field of $\sim 16 \mu \mathrm{G}$. No spectral analysis is performed, although the non-detection of the source by MAGIC and H.E.S.S. may imply a rather hard VHE gamma-ray spectrum.

\section{Relativistic outflows from gamma-ray binaries}

Gamma-ray binaries (GBs) have revealed in the last years as a new type of high-energy emitters. Being composed of a stellar-mass compact object orbiting a young, bright and massive star, GBs are observationally characterised by displaying the peak of their SED in the gamma-ray domain. As of today, eight systems have been robustly established as GBs: LS 5039 (Aharonian et al. 2005a), PSR B1259-63 (Aharonian et al. 2005b), LS I + 61303 (Albert et al. 2006), HESS J0632+057 (Aharonian et al. 2007), 1FGL J1018.6-5856 (Fermi LAT Collaboration et al. 2012), LMC P3 (Corbet et al. 2016), PSR J2032+4127 (Lyne et al. 2015) and, very recently, 4FGL J1405.1-6119 (Corbet et al. 2019). Amongst GBs, only in the cases of PSR B1259-63 and PSR J2032+4127 the compact object has been firmly identified as a neutron star, following the detection of pulsed emission at orbital phases in which the star is relatively far away from the free-free absorbing companion's stellar wind. The much higher compactness of other GBs prevents a similar detection of pulsations and therefore the identification of the nature of the compact object. Accretion-based models and pulsar-wind interaction scenarios keep being discussed as the powering engine of GBs (see e.g. Dubus 2006; Bosch-Ramon et al. 2006b; Romero et al. 2007; Torres 
et al. 2012; Jaron et al. 2016). An up-to-date summary accounting for some of the key properties of currently known GBs (not including 4FGL J1405.1-6119) is provided in (Paredes \& Bordas 2019a) and Paredes \& Bordas 2019b. For a more detailed review on GBs the reader is referred to (Dubus 2013).

GBs display a broad-band emission that extends from radio up to VHEs. On the one hand, leptonic mechanisms including synchrotron, Inverse Compton (IC) and thermal/relativistic Bremmstrahlung are extremely efficient radiative mechanisms in the framework of GBs given the extreme conditions of the matter, radiation and magnetic fields at binary-system length-scales. A magnetic field in the emitter zone can be provided by the plasma of the pulsar wind, or within the jet ejecta in the case of accretion-based models. The magnetic field associated to the stellar wind or to the external medium around the binary system can also act as a "seed" field for synchrotron to operate, but this requires the emitting particles to escape the pulsar wind or jet regions, e.g. in the forward shock at the jet/wind tip when the ejecta is halted by the swept-up external medium. IC emission is fed by the strong photon field provided by the companion star, typically a bright $\mathrm{O}$ or B spectral type star, or from the circumstellar disk present in some GBs. IC is however highly anisotropic (see e.g. Blumenthal \& Gould 1970), depending on the interaction angle between the electron and the seed photon. For an emitter located in the vicinity of the compact object this anisotropy will give rise to a periodically modulated light-curve, reflecting the changing interaction conditions along the orbital motion. Note, however, that photon-photon absorption can also be very efficient at VHEs. As $\gamma \gamma$ absorption is also highly anisotropic (see e.g. Dubus 2006), this modulation will also follow the orbital motion of the compact object around the companion star. Absorption will redistribute the energy flux towards lower energies. This can be used to constrain the location of the gamma-ray emission at or beyond binary-system length-scales in GBs (see e.g. Bosch-Ramon et al. 2008). Finally, the dense matter field in the close surroundings of gamma-ray binaries can make Bremsstrahlung emission and hadronic interactions an efficient emission mechanism as well. However, thermal Bremsstrahlung has not been detected yet in GBs (see e.g. Zabalza et al. 2011). Proton-proton interactions, on the other hand, which could also lead to the production of gamma-rays through $\pi^{0}$-decay, depend on the capabilities of GBs to accelerate protons to relativistic energies. Such scenario has been considered e.g. to explain the VHE gamma-ray emission from PSR B1259-63 (Neronov \& Chernyakova 2007). The predicted TeV profile, however, resulted to be markedly different from later observations of this source taken with the H.E.S.S. telescopes (H. E. S. S. Collaboration et al. 2013).

\subsection{Case study: PSR B1259-63}

PSR B1259-63 is a GB composed of a rotation-powered pulsar spinning every 48 ms originally detected as a radio pulsar (Johnston et al. 1992), and a Be companion star orbiting one each other every 3.4 years in a highly eccentric orbit with $e=0.87$ (see Negueruela et al. 2011 and references therein). The Be star features a dense equatorial outflow with a disk-like morphology. This disk is thought to be crossed twice per orbit by the neutron star at orbital phases close to periastron passage, when the source displays relatively high fluxes of non-thermal emission across the whole electromagnetic spectrum (see e.g. Chernyakova et al. 2014). The strong interaction between the stellar wind and the pulsar wind near the periastron passage has been shown to produce an outflow extending several AU away from the system (Moldón et al. 2011). The flux profile during 
these orbital phases features two distinct maxima roughly coincident in radio, X-rays and VHE gamma-rays, with the second peak displaying higher luminosities. At VHEs, the source has been monitored close to periastron since 2004 (Aharonian et al. 2005b, 2009; H. E. S. S. Collaboration et al. 2013). During the 2014 campaign, a third emission peak could be distinguished before the first disk crossing, although it is yet unclear if this is periodically repeating (Romoli et al. 2017).

At HE gamma-rays, PSR B1259-63 is also detected close to periastron passage, although the relative variability close to the disk-crossing times is weaker compared to radio, $\mathrm{X}$-rays and $\mathrm{TeV}$ gamma-rays. About 30-40 days after periastron, however, extremely bright gamma-ray flares have been observed with the Fermi-LAT in 2011, 2014 and 2017 (see e.g. Abdo et al. 2011; Caliandro et al. 2015; Tam et al. 2018; Chang et al. 2018). These flares displayed similar properties in terms of peak luminosities and onset times of about 30 days after periastron in 2011 and 2014. The total observed luminosity, adopting a distance to the source of $2.7 \mathrm{kpc}$ (Miller-Jones et al. 2018) strongly challenged current models on the source, as this was similar or higher than the total spin-down luminosity of the system $\left(\dot{E}=8.2 \times 10^{35} \mathrm{erg} \mathrm{s}^{-1}\right)$. Furthermore, the HE flare did not correspond to any strong enhancement of the non-thermal flux at any other wavelength (see e.g. H. E. S. S. Collaboration et al. 2013). During the 2017 periastron passage, the flare repeated again, although with some remarkable differences. The flare onset took place 40 days after periastron, lasting for about 30 days, and displayed higher fluxes and shorter variability, down to $\sim$ minute time-scales (Johnson et al. 2018).

These HE gamma-ray flares have been discussed in length since their discovery, although their origin remains unclear. A model accounting for IC emission from particles in the un-shocked pulsar wind were proposed by Khangulyan et al. (2012). This model can explain the narrow flare being detectable only at sub-GeV energies, with the timing of the flare being the result of pulsar wind zone increasing its size abruptly as the pulsar exits the disk-crossing phase. This model requires however an enhancement of the photon field upon which relativistic electrons in the un-shocked wind can scatter off, requiring an additional infrared photon field in order to reach the observed luminosities in the flare time-scales. IC models have also been invoked considering a narrow energy distribution of electron/positron pairs produced close to the pulsar and scattering off X-ray photons generated in the tail of the shocked pulsar wind (Dubus \& Cerutti 2013). Doppler-boosted synchrotron emission has also been invoked to model the HE outbursts (see e.g. Kong et al. 2012). Doppler effects can indeed help to explain the high flare luminosities observed, although such an enhancement should also be detected at other wavelengths, which is not the case. For the 2017 flare, displaying the most extreme properties in terms of luminosity and variability, IC models seem to be put at their limits, whereas Doppler factors of at least $D \gtrsim 3$ and a rather high electron acceleration efficiency (Kong et al. 2012) are required in synchrotron-based models (Johnson et al. 2018). Variability down to $\sim$ minute time-scales requires the emission region to be $\sim 30 \%-40 \%$ of the separation distance between the pulsar and the Be companion star at the time of the flare (Johnson et al. 2018). Far away from periastron passage, $X$-ray extended emission has been reported from PSR B1259-63 using Chandra observations of the source close to apastron orbital phases (Pavlov et al. 2011). This emission has been interpreted as synchrotron emission from the pulsar wind when it is shocked by a clump of plasma ejected from PSR B1259-63 at the time of the system periastron passage (Pavlov et al. 2015; see also Barkov \& Bosch-Ramon 2016). It is unclear whether the strong HE flares are related to the production of this ejecta. 


\section{Jet-like features in runaway pulsars}

Pulsars are amongst the most powerful and efficient particle accelerators known in Nature. Their huge rotational energy is dissipated away in the form of a highly relativistic wind, which is observed to radiate non-thermally in the whole electromagnetic spectrum up to energies that can reach tens of TeV (see, e.g. H. E. S. S. Collaboration et al. 2019a; H. E. S. S. Collaboration et al. 2019b). The integrated emission from these winds can actually be several orders of magnitude brighter than the pulsar magnetosphere itself. This emission is thought to be produced through synchrotron radiation by relativistic particles and magnetic fields present in the wind, together with IC emission by this very same particle population scattering off external photon fields (Cosmic Microwave Background radiation, dust IR, and starlight photons). In the case of runaway pulsars moving at supersonic velocities in the interstellar medium (ISM), this wind displays a bullet-like structure when the wind inertia is balanced by the ram pressure of the surrounding medium (see e.g. (Gaensler et al. 2004); see also the detailed MHD simulations of runaway PWN morphologies in Bucciantini et al. (2005). A bow shock is formed ahead of the pulsar, at a distance $R_{\mathrm{bs}} \sim$ $\left(\dot{E} / 4 \pi c P_{\text {ISM }}\right)^{1 / 2}$, where $\dot{E}$ is the pulsar spin-down power and $P_{\text {ISM }}$ is the ambient medium thermal pressure. Long cometary tails filled with the shocked pulsar wind material are formed behind the pulsar motion, and in some cases striking structures displaying long jet-like features that can extend for more than $10 \mathrm{pc}$ into the surrounding medium have been resolved, in particular at $\mathrm{X}$ rays thanks to the good performance of instruments like XMM-Newton and Chandra in terms of angular resolution (see Kargaltsev et al. 2017 for a review; see also Fig. 4 for one of the most extreme examples, the system IGR J11014-6103).

The study of pulsar wind nebulae (PWNe) can provide invaluable information about the central engines powering them, the properties of the surrounding medium, and the physical processes involved in the wind-medium interactions. In the case of runaway PWNe, the structures formed by the shocked pulsar wind are put at their limits. In this case, wind particles at the termination shock may undergo re-acceleration in between the forward shock (into the medium) and the reverse shock (a shock within the pulsar wind itself). If magnetic perturbations are accounted for, e.g. produced by the same accelerated particles, this re-acceleration can lead to hard particle distributions, which would explain the rather hard X-ray spectra generally retrieved for runaway PWNs. These reaccelerated particles, could contribute a substantial fraction of the positrons detected as cosmic-ray particles above a few tens of $\mathrm{GeV}$ and up to several hundred $\mathrm{GeV}$ (see e.g. Bykov et al. 2017). There are about 30 of such runaway PWN identified so far through observations in X-rays that are able to reveal their unique morphologies, through $H_{\alpha}$ emission lines identifying the shocked structures, through observations in the far-UV, which have resolved the bow shock in a couple of systems thanks to the capabilities of the Hubble Space Telescope, and through radio observations, revealing to lower-energy synchrotron emission from about 8 runaway PWNe (Kargaltsev et al. 2017),

\subsection{Case study: IGR J11014-6103}

In addition to the strong shocks ahead of runaway PWNe and the tail-like structures behind them, recent X-ray observations have also revelaed parsec-scale jet-like features in some of the highest velocity pulsars. These structures can develop almost perpendicularly to their direction of 


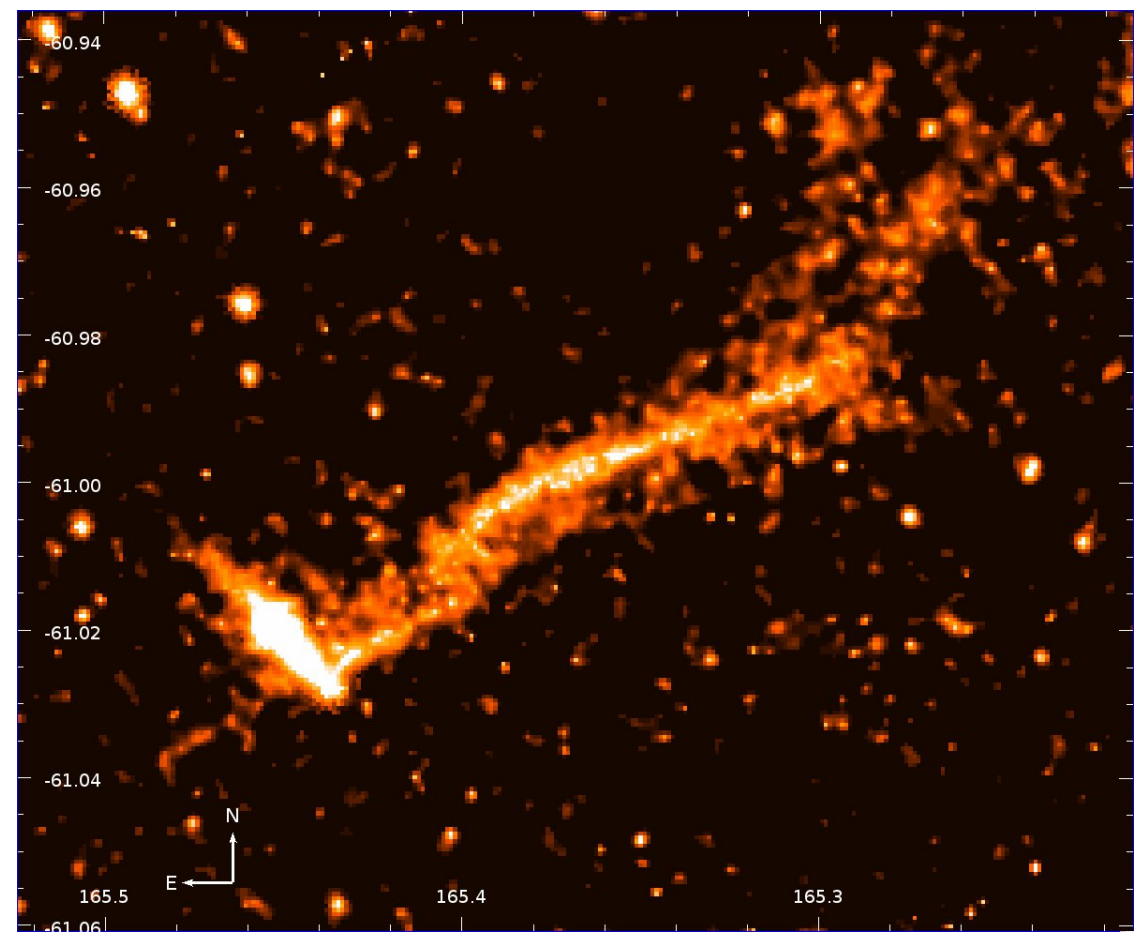

Figure 1: Flux map around IGR J11014-6103 obtained with Chandra (adapted from Pavan et al. 2014). Besides the pulsar and the nebular tail emission, a prominent jet-like feature propagating along the northwest direction is clearly visible. A shorter, rectilinear counter-jet feature, propagating in the south-east direction, can also be distinguished.

motion, without any clear/strong signature of bending. Although several hypotheses have been explored, at present the launching and propagation mechanisms of these rare outflows is still unclear. The source IIGR J11014-6103 is the most extreme case known so far, hosting the brightest and most elongated jet-like features known so far in our Galaxy (see e.g. Pavan et al. 2014).

IGR J11014-6103 is located 11 arcmin south-west of the SNR MSH 11-61A. In soft X-rays (1-10 keV) the system is observed to contain a pulsar (PSR J1101-6101) expelled by its parent SNR MSH 11-61A, a runaway PWN trailing back along the direction of motion in direction of the parent SNR, and long and collimated X-ray jet-like structure extending perpendicularly over 7-arcmin (Pavan et al. 2011). The association of IGR J11014-6103 to SNR MSH 11-61A lead to an estimated projected speed of more than $1000 \mathrm{~km} / \mathrm{s}$ for the pulsar which is translated to about $15 \mathrm{pc}$ for the physical elongation of the jet. This is the longest X-ray jet-like feature ever observed in our Galaxy. Observations with the Chandra satellite revealed in addition a striking "S-shape" modulation of the longest outflow north of IGR J11014-6103. The short length-scale and regularity of the modulation is strongly reminiscent of a precession or kink instability pattern, suggesting a possible ballistic jet interpretation for this structure (Pavan et al. 2014, 2016). However, the almost unbent shape of the outflow, accounting for the high-velocity of the system, severely challenges this interpretation. In this regard, other scenarios have been suggested for other runaway PWNe displaying similarly misaligned jet-like features, in particular a scenario in which these features are 
produced through electrons accelerated at the bow-shock interface that diffuse away following the underlying structure of the medium magnetic field (see e.g. Bandiera 2008).

In this diffusion scenario, accelerated electrons are able to escape the bow-shock region once their gyro-radius $r_{\mathrm{g}}=\gamma m_{\mathrm{e}} c^{2} / e B$ becomes larger than the stand-off distance of the bow-shock apex ( $\sim R_{\mathrm{bs}}$, as defined above). These particles cannot be contained within the bow-shock and can therefore leak into the ISM and diffuse along the ambient magnetic field lines and radiate synchrotron photons. This escape/diffusion scenario has been further studied through MHD simulations recently (see e.g. Barkov et al. 2019; Olmi \& Bucciantini 2019), which have confirmed its viability and even explained the circumstances under which highly asymmetric counter-outflows can be produced in runaway PWNe). Recent observations of IGR J11014-6103, however, seem to partially challenge these results, as the misaligned outflow appears to bend around the pulsar position, after which it becomes much more rectilinear. Although one could argue that for such a fast-moving system the distortion of the ISM magnetic field structure is rather expected, such influence should be contained within the shocked medium region (Lyutikov 2006; Dursi \& Pfrommer 2008). A number of questions regarding runaway PWN remain in addition unresolved. From a morphological perspective, it is yet unclear which are the physical conditions upon which the relative prominence of tails and jet-like features depend for a given system/environment. From an observational perspective, to date there is no single runaway PWN detected at TeV gamma-rays, whereas low-velocity PWN are some of the best studied populations at these energies. The lack of $\mathrm{TeV}$ detections has been suggested to be related to the limitations of current VHE gamma-ray observatories in terms of angular resolution, such that they may not be able resolve the narrow and long structures associated to runaway PWN (Kargaltsev et al. 2017). However, it is worth noting that non-resolving these structures does not imply a non-detection of these systems, which otherwise display spindown powers as high as $\sim 10^{37} \mathrm{erg} \mathrm{s}^{-1}$.

\section{Concluding remarks}

The study of high-energy phenomena associated to relativistic outflows from galactic sources has provided in the last years a number of discoveries which defer further study for their understanding. In this contribution we briefly reviewed three of such cases, namely galactic microquasars, gamma-ray binaries, and runaway pulsar wind nebulae, focusing on recent findings obtained for the corresponding sources SS433, PSR B1259-63 and IGR J11014-6103 . For the former case, several groups have reported in this last years the detection of the source at HE gamma-rays. The origin of this emission is still highly debated in terms of its localisation or whether it comes from the central system or from the eastern/western lobes. The hints for precession periodicity reported in Rasul et al. (2019) would imply that at least a fraction of the gamma-ray flux may be originated at regions relatively close to the central system where this modulation is effective - periodic precession signatures may be smeared out at larger distances unless exotic scenarios are invoked. On spectral grounds, the narrowly peaked SED reported in (Bordas et al. 2015) (see also Rasul et al. 2019) seems to favour hadronic models for this emission. At VHEs, however, the HAWC collaboration has recently reported the detection of SS433/W50 at energies above $\sim 25 \mathrm{TeV}$, which may be produced in the eastern/western lobes, not from the central system. Furthermore, these authors favour a leptonic origin of the emission. It is worth noting that this model fails in accounting 
simultaneously for both the HE and VHE gamma-ray emission from the source (see e.g. Xing et al. 2019). As for GBs, the number of systems of this class is slowly increasing, with 8 sources firmly detected at gamma-rays as of today. The powering engine in most cases remains unknown, with the exception of PSR B1259-63 and PSR J2032+4127, for which the nature of the compact object, a neutron star, has been unambiguously identified. In the case of PSR B1259-63, the monitoring of three periastron passages of the system with current HE gamma-ray instruments has shown the repeated occurrence of bright HE flares which carry a significant fraction of the system energy reservoir. In some instances, particularly for the flare observed in 2017 and for the analysis of short time-scale data-sets, the total luminosity clearly exceeds the pulsar spin-down power. Although several models have been proposed none of them can explain the flares consistently in a broadband MWL framework. Regarding runaway PWN, recent studies have demonstrated these sources being capable of producing some of the most extreme jet-like structures known in the Galaxy. The origin of these structures is not yet clear, although recent MHD simulations seem to confirm the possibility of high-energy particle diffusion into the ambient magnetic field. Interestingly, this diffusion scenario, alternative to the more traditional one based on "ballistic" jets, may also explain the radio filaments in the central regions of our Galaxy (see e.g. Barkov \& Lyutikov (2019).

\section{References}

Abdo, A. A., Ackermann, M., Ajello, M., et al. 2011, ApJL, 736, L11

Abell, G. O. \& Margon, B. 1979, Nature, 279, 701

Abeysekara, A. U., Albert, A., Alfaro, R., et al. 2018, Nature, 562, 82

Aharonian, F., Akhperjanian, A. G., Anton, G., et al. 2009, A\&A, 507, 389

Aharonian, F., Akhperjanian, A. G., Aye, K.-M., et al. 2005a, Science, 309, 746

Aharonian, F., Akhperjanian, A. G., Aye, K.-M., et al. 2005b, A\&A, 442, 1

Aharonian, F. A., Akhperjanian, A. G., Bazer-Bachi, A. R., et al. 2007, A\&A, 469, L1

Aharonian, F. A. \& Atoyan, A. M. 1998, NewAR, 42, 579

Albert, J., Aliu, E., Anderhub, H., et al. 2006, Science, 312, 1771

Albert, J., Aliu, E., Anderhub, H., et al. 2007, ApJl, 665, L51

Aleksić, J., Antonelli, L. A., Antoranz, P., et al. 2010, ApJ, 721, 843

Archambault, S., Beilicke, M., Benbow, W., et al. 2013, ApJ, 779, 150

Bandiera, R. 2008, A\&A, 490, L3

Barkov, M. V. \& Bosch-Ramon, V. 2016, MNRAS, 456, L64

Barkov, M. V. \& Lyutikov, M. 2019, MNRAS, 489, L28 
Barkov, M. V., Lyutikov, M., Klingler, N., \& Bordas, P. 2019, MNRAS, 485, 2041

Blumenthal, G. R. \& Gould, R. J. 1970, Reviews of Modern Physics, 42, 237

Bordas, P. 2009, PhD thesis, -

Bordas, P., Bosch-Ramon, V., \& Paredes, J. M. 2010, International Journal of Modern Physics D, 19,749

Bordas, P., Bosch-Ramon, V., Paredes, J. M., \& Perucho, M. 2009, A\&A, 497, 325

Bordas, P., Sun, X., Yang, R., Kafexhiu, E., \& Aharonian, F. A. 2017, in American Institute of Physics Conference Series, Vol. 1792, 6th International Symposium on High Energy GammaRay Astronomy, 040020

Bordas, P., Yang, R., Kafexhiu, E., \& Aharonian, F. 2015, ApJl, 807, L8

Bosch-Ramon, V., Aharonian, F. A., \& Paredes, J. M. 2005, A\&A, 432, 609

Bosch-Ramon, V., Khangulyan, D., \& Aharonian, F. A. 2008, A\&A, 489, L21

Bosch-Ramon, V., Romero, G. E., \& Paredes, J. M. 2006a, A\&A, 447, 263

Bosch-Ramon, V., Romero, G. E., \& Paredes, J. M. 2006b, A\&A, 447, 263

Bucciantini, N., Amato, E., \& Del Zanna, L. 2005, A\&A, 434, 189

Bykov, A. M., Amato, E., Petrov, A. E., Krassilchtchikov, A. M., \& Levenfish, K. P. 2017, SSR, 207,235

Caliandro, G. A., Cheung, C. C., Li, J., et al. 2015, ApJ, 811, 68

Chang, Z., Zhang, S., Chen, Y.-P., et al. 2018, Research in Astronomy and Astrophysics, 18, 152

Chardin, G. \& Gerbier, G. 1989, A\&A, 210, 52

Chernyakova, M., Abdo, A. A., Neronov, A., et al. 2014, MNRAS, 439, 432

Corbet, R. H. D., Chomiuk, L., Coe, M. J., et al. 2019, ApJ, 884, 93

Corbet, R. H. D., Chomiuk, L., Coe, M. J., et al. 2016, ApJ, 829, 105

Dermer, C. D. \& Böttcher, M. 2006, ApJ, 643, 1081

Díaz Trigo, M., Migliari, S., Miller-Jones, J. C. A., \& Guainazzi, M. 2014, A\&A, 571, A76

Díaz Trigo, M., Miller-Jones, J. C. A., Migliari, S., Broderick, J. W., \& Tzioumis, T. 2013, Nature, 504,260

Dubner, G. M., Holdaway, M., Goss, W. M., \& Mirabel, I. F. 1998, AJ, 116, 1842

Dubus, G. 2006, A\&A, 456, 801 
Dubus, G. 2013, A\&Ar, 21, 64

Dubus, G. \& Cerutti, B. 2013, A\&A, 557, A127

Dursi, L. J. \& Pfrommer, C. 2008, ApJ, 677, 993

Fermi LAT Collaboration, Abdo, A. A., Ackermann, M., et al. 2009, Science, 326, 1512

Fermi LAT Collaboration, Ackermann, M., Ajello, M., et al. 2012, Science, 335, 189

Gaensler, B. M., van der Swaluw, E., Camilo, F., et al. 2004, ApJ, 616, 383

H. E. S. S. Collaboration, Abdalla, H., Abramowski, A., et al. 2018, A\&A, 612, A10

H. E. S. S. Collaboration, Abdalla, H., Aharonian, F., et al. 2019a, A\&A, 621, A116

H. E. S. S. Collaboration, Abdalla, H., Aharonian, F., et al. 2019b, A\&A, 627, A100

H. E. S. S. Collaboration, Abramowski, A., Acero, F., et al. 2013, A\&A, 551, A94

Heinz, S. \& Sunyaev, R. 2002, A\&A, 390, 751

Jaron, F., Torricelli-Ciamponi, G., \& Massi, M. 2016, A\&A, 595, A92

Johnson, T. J., Wood, K. S., Kerr, M., et al. 2018, ApJ, 863, 27

Johnston, S., Manchester, R. N., Lyne, A. G., et al. 1992, ApJL, 387, L37

Kargaltsev, O., Pavlov, G. G., Klingler, N., \& Rangelov, B. 2017, Journal of Plasma Physics, 83, 635830501

Khangulyan, D., Aharonian, F. A., Bogovalov, S. V., \& Ribó, M. 2012, ApJ1, 752, L17

Kong, S. W., Cheng, K. S., \& Huang, Y. F. 2012, ApJ, 753, 127

Loh, A., Corbel, S., Dubus, G., et al. 2016, MNRAS, 462, L111

Lyne, A. G., Stappers, B. W., Keith, M. J., et al. 2015, MNRAS, 451, 581

Lyutikov, M. 2006, MNRAS, 373, 73

MAGIC Collaboration, Ahnen, M. L., Ansoldi, S., et al. 2018, A\&A, 612, A14

Malyshev, D., Zdziarski, A. A., \& Chernyakova, M. 2013, MNRAS, 434, 2380

MeerKAT Collaboration. 2018, MNASSA : Monthly Notes of the Astronomical Society of South Africa, 77, 102

Migliari, S., Fender, R., \& Méndez, M. 2002, Science, 297, 1673

Miller-Jones, J. C. A., Deller, A. T., Shannon, R. M., et al. 2018, MNRAS, 479, 4849

Mirabel, I. F., Rodriguez, L. F., Cordier, B., Paul, J., \& Lebrun, F. 1992, Nature, 358, 215 
Moldón, J., Johnston, S., Ribó, M., Paredes, J. M., \& Deller, A. T. 2011, ApJL, 732, L10

Negueruela, I., Ribó, M., Herrero, A., et al. 2011, ApJL, 732, L11

Neronov, A. \& Chernyakova, M. 2007, ApSS, 309, 253

Olmi, B. \& Bucciantini, N. 2019, MNRAS, 488, 5690

Paredes, J. M. \& Bordas, P. 2019a, arXiv e-prints, arXiv:1901.03624

Paredes, J. M. \& Bordas, P. 2019b, arXiv e-prints, arXiv:1902.09898

Pavan, L., Bordas, P., Pühlhofer, G., et al. 2014, A\&A, 562, A122

Pavan, L., Bozzo, E., Pühlhofer, G., et al. 2011, A\&A, 533, A74

Pavan, L., Pühlhofer, G., Bordas, P., et al. 2016, A\&A, 591, A91

Pavlov, G. G., Chang, C., \& Kargaltsev, O. 2011, ApJ, 730, 2

Pavlov, G. G., Hare, J., Kargaltsev, O., Rangelov, B., \& Durant, M. 2015, ApJ, 806, 192

Rasul, K., Chadwick, P. M., Graham, J. A., \& Brown, A. M. 2019, MNRAS, 485, 2970

Reynoso, M. M., Christiansen, H. R., \& Romero, G. E. 2008, Astroparticle Physics, 28, 565

Romero, G. E., Okazaki, A. T., Orellana, M., \& Owocki, S. P. 2007, A\&A, 474, 15

Romero, G. E., Torres, D. F., Kaufman Bernadó, M. M., \& Mirabel, I. F. 2003, A\&A, 410, L1

Romoli, C., Bordas, P., Mariaud, C., Murach, T., \& H. E. S. S. Collaboration. 2017, in International Cosmic Ray Conference, Vol. 301, 35th International Cosmic Ray Conference (ICRC2017), 675

Sabatini, S., Tavani, M., Coppi, P., et al. 2013, ApJ, 766, 83

Safi-Harb, S. \& Ögelman, H. 1997, ApJ, 483, 868

Saito, T. Y., Zanin, R., Bordas, P., et al. 2009, arXiv e-prints, arXiv:0907.1017

Tam, P.-H. T., He, X., Sarathi Pal, P., \& Cui, Y. 2018, ArXiv e-prints

Tavani, M., Bulgarelli, A., Piano, G., et al. 2009, Nature, 462, 620

Torres, D. F., Rea, N., Esposito, P., et al. 2012, ApJ, 744, 106

Xing, Y., Wang, Z., Zhang, X., Chen, Y., \& Jithesh, V. 2019, ApJ, 872, 25

Zabalza, V., Bosch-Ramon, V., \& Paredes, J. M. 2011, ApJ, 743, 7

Zanin, R., Fernández-Barral, A., de Oña Wilhelmi, E., et al. 2016, A\&A, 596, A55

Zdziarski, A. A., Malyshev, D., Chernyakova, M., \& Pooley, G. G. 2017, MNRAS, 471, 3657 